\title{
Fragilidades da passagem de plantão em uma unidade pediátrica na perspectiva da equipe de enfermagem
}

\author{
Fragility of the shift in a pediatric unit from the perspective of the nursing team \\ Fragilidades del paso de turno en una unidad pediátrica en la perspectiva del equipo de enfermería \\ Michelini Fátima da Silva ${ }^{1}$, Jane Cristina Anders ${ }^{1}$, Patrícia Kuerten Rocha ${ }^{1}$, Andréia Tomazoni ${ }^{2}$, Soliane Quitolina Scapin ${ }^{2}$
}

\section{Resumo}

Objetivo: Identificar os fatores que interferem na comunicação durante a passagem de plantão e que repercutem na segurança do paciente pediátrico.

Métodos: Dados coletados em fevereiro e maio de 2012, através de entrevista semiestruturada com 32 profissionais de enfermagem.

Resultados: Originou-se uma categoria: "Os fatores que interferem na comunicação: transcender barreiras para conduzir a passagem de plantão", demonstrando que a comunicação eficaz na passagem de plantão requer a superação das barreiras de ordem física e humana.

Conclusão: Diante da magnitude e da complexidade que envolve a segurança do paciente pediátrico, não basta que os profissionais se preocupem com a utilização de recursos tecnológicos e com o aprimoramento de técnicas, mas devem também estar atentos às habilidades e competências para realizar uma comunicação eficaz na passagem de plantão.

\section{Abstract}

Objective: To identify the factors that interfere in the communication during the shift and that have repercussions on the safety of the pediatric patient.

Methods: Data collected in February and May 2012, through a semi-structured interview with 32 nursing professionals.

Results: A category arose: "The factors that interfere in communication: transcending barriers to conduct the shift," demonstrating that effective communication at the shift requires the overcoming of physical and human barriers." Conclusion: Given the magnitude and complexity of pediatric patient safety, it is not enough that professionals are concerned with the use of technological resources and with the improvement of techniques; they should be aware of the skills and competencies to perform effective communication on shift duty.

\section{Resumen}

Objetivo: Identificar los factores que interfieren en la comunicación durante el paso de turno y que repercuten en la seguridad del paciente pediátrico.

Métodos: Datos recogidos en febrero y mayo de 2012, a través de una entrevista semiestructurada con 32 profesionales de enfermería.

Resultados: Se originó una categoría: "Los factores que interfieren en la comunicación: trascender barreras para conducir el paso de turno", demostrando que la comunicación eficaz en el paso de turno requiere la superación de las barreras de orden físico y humano

Conclusión: Ante la magnitud y la complejidad que implica la seguridad del paciente pediátrico, no basta que los profesionales se preocupen por la utilización de recursos tecnológicos y con el perfeccionamiento de técnicas; ellos deben también estar atentos a las habilidades y competencias para realizar una comunicación eficaz en el paso de turno.

\section{Descritores}

Comunicação; Segurança; Enfermagem pediátrica

Keywords

Communication; Safety; Pediatric nursing

\section{Descriptores}

Comunicación; Seguridad; Enfermería pediátrica

\section{Como citar:}

Silva MF, Anders JC, Rocha PK, Tomazoni A, Scapin SQ. [Fragility of the shift in a pediatric unit from the perspective of the nursing team]. Rev Soc Bras Enferm Ped. 2018;18(2):62-8. Portuguese

1 Universidade Federal de Santa Catarina, Florianópolis, SC, Brasil.

2 Hospital de Clínicas, Universidade Federal do Paraná, Curitiba, PR, Brasil.

Conflitos de interesse: nada a declarar.

Submissão: 21 de Junho de 2016 | Aceite: 27 de Junho de 2019

Autor correspondente: Michelini Fátima da Silva | Rua Professora Maria Flora Pausewang, s/nº, 88036-800, Florianópolis, SC, Brasil. michelinisilva@yahoo.com.br https://orcid.org/0000-0002-2891-0327

DOI: http://dx.doi.org/10.31508/1676-3793201800010 


\section{Introdução}

Nos últimos anos, as organizações e agências internacionais de saúde têm evidenciado a necessidade de desenvolverem metas e ações para melhorar a segurança do paciente. Ao longo desse período foram desenvolvidas um número significativo de estratégias voltadas à qualidade do cuidado, à saúde e, consequentemente, diminuir os riscos relacionados à assistência a saúde. ${ }^{(1)}$

$O$ cuidado de enfermagem seguro exige conhecimento, habilidade, responsabilidade e compromisso. Com isso, parece óbvio que a qualidade do cuidado e a segurança do paciente hospitalizado são metas a serem atingidas pelos profissionais e instituições de saúde. No entanto, apesar de esforços no sentido de alcançar um cuidado qualificado, humanizado, livre de riscos potenciais e iatrogenias, alicerçado pelo desenvolvimento tecnológico e científico, constatam-se ainda inúmeras ocorrências de eventos adversos durante a hospitalização dos pacientes. ${ }^{(2)}$

Esses eventos originam-se de uma gama de fatores relacionados tanto às questões profissionais como institucionais, repercutindo na segurança do paciente. Dentre os fatores desencadeadores de erros e eventos adversos, a falha no processo de comunicação durante a passagem de plantão tem contribuído amplamente para a ocorrência desses.

A passagem de plantão é uma atividade variável e propensa a erros, os fatores ambientais como ruídos, aglomeração de pessoas e interrupções, são potenciais ameaças à sua qualidade. Desta forma, tem sido considerada internacionalmente como uma área de alto risco para a segurança do paciente. Em decorrência dessa situação, a comunicação tem sido discutida, em âmbito nacional e internacional, por instituições e organizações de saúde, com o objetivo de estabelecer iniciativas que visam à melhoria das passagens de plantão. ${ }^{(3)}$

A prevenção de erros durante a passagem de plantão é uma das cinco áreas de soluções da "High $5 \mathrm{~s}$ initiative", um mecanismo estabelecido em 2006 por meio da colaboração entre o Commonwealth Fund, a WHO World Alliance for Patient Safety e a WHO Collaborating Centre for Patient Safety, para implementar soluções inovadoras de segurança do paciente ao longo dos próximos cinco anos. ${ }^{(1)}$

Em 2007, a Aliança Mundial para a Segurança do Paciente da Organização Mundial da Saúde (OMS), em parceria com outros países, definiu algumas metas para a segurança do paciente, entre as quais está a comunicação efetiva na passagem de plantão. ${ }^{(4)}$

Atualmente, buscam-se experiências bem-sucedidas que promovam a segurança do paciente no sistema de saúde, analisando atribuições e responsabilidades dos profissionais para o gerenciamento de riscos em saúde e a promoção da qualidade da segurança. ${ }^{(5)}$

Avaliando as metas estabelecidas pela The Joint Commission percebe-se que todas se concentram em áreas de grande importância para a segurança dos pacientes no meio hospitalar. No entanto, optou-se por abordar a comunicação eficaz, por esta ser essencial durante a comunicação, na passagem de plantão. A efetividade da comunicação nas instituições de saúde reduz a ocorrência de erros e resulta na melhora da segurança do paciente.

A escolha por este tema como objeto de pesquisa além do que já foi citado, se deu também por perceber, diante de experiências profissionais que, os fatores que interferem a comunicação na passagem de plantão são alarmantes, estudos demonstram que deficiências na comunicação o durante a passagem de plantão têm sido um dos principais fatores que contribuem para a ocorrência de erros, eventos adversos (EAs) e, consequentemente, diminuição da qualidade da assistência.

Diante do contexto ora exposto, e considerando a importância do tema, os esforços globais em inserir medidas de prevenção e, a importância em investir em estudos que contribuam para comunicação efetiva durante a passagem de plantão surgiu o seguinte questionamento: Quais os fatores que interferem na comunicação durante a passagem de plantão e que repercutem na segurança do paciente pediátrico?

\section{Métodos}

Estudo descritivo-exploratório, de natureza qualitativa, realizado em um hospital de médio porte em Florianópolis, Brasil na Unidade de Internação Pediátrica (UIP). Participaram da pesquisa 32 profissionais da equipe de enfermagem, sendo sete enfermeiras, 14 técnicos e 11 auxiliares de enfermagem que atuam na UIP.

A coleta de dados empíricos foi realizada durante os meses de fevereiro à maio de 2012. Foi utilizada como fonte para a coleta dos dados a entrevista se- 
miestruturada, gravada após a permissão dos profissionais da equipe de enfermagem. Cabe ressaltar que todos os participantes assinaram o Termo de Consentimento Livre e Esclarecido.

Além disso, durante a realização da entrevista, questionamentos acerca da qualidade e fatores que interferem na passagem de plantão foram levantados. Sobretudo, sabe-se que a passagem de plantão tem como finalidade a transmissão de informações referentes à assistência prestada à criança, adolescente e seus familiares, sendo realizada, neste setor (UIP), na sala de chefia de enfermagem, conduzida pelos enfermeiros assistenciais com a participação da equipe de enfermagem e alunos dos diversos cursos da área da saúde. Ainda, ressalta-se que acontece em três turnos (manhã, tarde e noite) ao final do expediente de cada.

A entrevista seguiu um roteiro constando de duas partes: a primeira - identificação dos participantes - aborda questões relacionadas a idade, sexo, escolaridade, categoria profissional, tempo que trabalha na instituição e número de vínculos empregatícios. Já a segunda parte é composta de cinco questões norteadoras sendo elas: 1-Você considera a comunicação uma ferramenta importante para a passagem de plantão? Conte como acontece a passagem de plantão na UIP. 2- Fale um pouco sobre os fatores que interferem na comunicação durante a passagem de plantão. 3- Você acha a passagem de plantão importante para a continuidade do cuidado de enfermagem e para a segurança da criança e do adolescente hospitalizado? O que leva você a ter essa opinião? 4- Quais as informações que são importantes e devem ser comunicadas durante a passagem de plantão? 5- Você teria alguma sugestão para melhorar a comunicação durante a passagem de plantão visando à segurança do paciente pediátrico?

Para assegurar a privacidade e o sigilo dos dados, utilizou-se um sistema de identificação no qual os nomes verdadeiros foram substituídos pela letra "E" para as enfermeiras, letra " $\mathrm{T}$ " para os técnicos de enfermagem e letra " $\mathrm{A}$ " para os auxiliares de enfermagem, seguida de um algarismo numérico para diferenciá-las entre si. Por exemplo: E1 (enfermeira 1), E2 (enfermeira 2), e assim sucessivamente.

Para a análise dos dados foi utilizado a Análise Temática, que consiste em pré-análise, na qual se procedeu à organização dos dados coletados nas entrevis- tas, seguida da exploração desse material, considerada a análise propriamente dita que permitiu codificar os dados e formular a categoria e subcategoria através da identificação de conceitos pertinentes aos elementos e às ideias do estudo proposto. Por último, realizou-se a etapa de tratamento e interpretação dos resultados. ${ }^{(6)}$

Cabe ressaltar que este projeto respeitou todas as normas de pesquisa com seres humanos e foi submetido à apreciação do Comitê de Ética em Pesquisa da referida instituição, sendo aprovado sob o parecer no 2232 .

\section{Resultados}

Dos profissionais entrevistados 29 eram do sexo feminino, com idade entre 23 e 61 anos e três do sexo masculino, com idade entre 33 e 49 anos. O tempo de atuação na UIP variou entre um a 20 anos. Em relação ao vínculo empregatício nove profissionais possuíam mais de um vínculo. Quanto ao nível de escolaridade cinco profissionais possuíam curso de especialização, sete curso de mestrado e um de doutorado. A partir das entrevistas foi possível a apreensão da categoria " $\mathrm{Fa}$ tores que interferem na comunicação: transcender barreiras para conduzir a passagem de plantão", da qual se extraíram duas subcategorias: "barreiras relacionadas ao ambiente de trabalho" e "barreiras relacionadas à participação dos funcionários na passagem de plantão".

\section{Barreiras relacionadas ao ambiente de trabalho na passagem de plantão}

Para os profissionais entrevistados as barreiras referentes ao ambiente de trabalho estão diretamente relacionadas à sua organização. Os mesmos também percebem o local onde é realizada essa atividade como um espaço que interfere na comunicação eficaz e consequentemente na passagem de plantão.

[...] o local não é adequado, espaço muito pequeno, poucos lugares para sentar, sala muito no meio da unidade. (E2)

O local não é adequado tinha que ser mais amplo, mais arejado, são muitas pessoas na mesma sala, fica tudo amontoadinho. (A1)

Outro fator evidenciado nas entrevistas está relacionado ao impacto dos aparelhos eletrônicos (televisão, telefone e computador) ligados durante a passagem de plantão, ocasionando distração dos pro- 
fissionais, conforme podemos evidenciar nos relatos a seguir:

O telefone ou o celular às vezes toca, tirando a concentração de todos. (A5)

A televisão ligada tira a atenção da gente. (T9)

[...] o computador ligado atrapalha. (E4)

Para os entrevistados, as interrupções realizadas pelos acompanhantes e/ou familiares das crianças e adolescentes hospitalizados são fatores que interferem na passagem de plantão, como se pode verificar nos relatos:

Os pais vem pedir algo, sempre quando estamos passando o plantão. (A6)

É impressionante mas sempre na passagem vem algum acompanhante pedir alguma coisa [...] muitas vezes não é urgente e poderia esperar terminar a passagem de plantão. (E3)

\section{Barreiras relacionadas à participação dos funcionários na passagem de plantão}

Outros fatores apontados pelos profissionais de enfermagem relacionam-se ao comprometimento profissional e à responsabilidade ética. Ao serem indagados sobre os fatores que interferem na comunicação, os entrevistados trouxeram exemplos vivenciados na prática profissional, fazendo menção ao descomprometimento de alguns profissionais em relação à passagem de plantão. Algumas falas revelam essa situação:

[...] muitos funcionários chegam atrasados. (A3)

A maioria dos funcionários vai embora para não chegar atrasado no outro hospital. (A6)

A passagem de plantão não é padronizada e nem está amparada em qualquer lei relacionada ao exercício profissional ou ao contrato de trabalho, mas é amplamente cobrada no momento da execução das atividades.

[...] A passagem de plantão não é só para a enfermeira [..]. (E5)

[...] Os técnicos e auxiliares precisam estar presentes. (T8)

Muitos funcionários não querem nem saber, simplesmente vão embora. (A7)

Já procurei na lei do exercício profissional algo que falasse que eu sou obrigada a passar plantão e não encontrei nada. (T5)

Ainda, quanto ao comprometimento, destacou-se ainda a falta de atenção no momento da passagem de plantão:
[...] As pessoas às vezes não prestam a atenção. (T3)

Existe muita conversa paralela durante a passagem de plantão. (E6)

Não se tem ainda o respeito pelo ritual da passagem de plantão da forma como deveria ser. (E6)

\section{DISCUSSÃO}

\section{Barreiras relacionadas ao ambiente de trabalho na passagem de plantão}

As barreiras são os fatores que dificultam ou interrompem a comunicação e podem ser originadas por diferenças ambientais, culturais, sociais, intelectuais e éticas. Ao reconhecer e utilizar medidas preventivas para essas barreiras, os profissionais de enfermagem podem se comunicar de forma eficaz.

As barreiras de segurança são ações que visam diminuir fatores de risco que envolvem o processo de assistência em saúde, na tentativa de impedir a ocorrência de eventos adversos. Essas devem ser exploradas para posteriormente se estabelecerem medidas preventivas que melhoram a segurança do paciente. ${ }^{(7)}$

O espaço físico se refere à estrutura em si e seus complementos, como: ventilação, móveis e materiais. $\mathrm{O}$ ambiente psicossocial inclui a cultura organizacional, bem como atitudes, valores, crenças e práticas cotidianas das instituições. ${ }^{(8)} \mathrm{O}$ ambiente de trabalho tem grande influência na prática da enfermagem, tanto na comunicação, como na segurança e na satisfação profissional. ${ }^{(9)}$

O ambiente superlotado prejudica a concentração na atividade e interfere na comunicação durante a passagem de plantão. Rotineiramente, os plantões diurnos tendem a ser mais atribulados, com a presença de um maior número de profissionais da equipe de enfermagem. Além disso, a unidade de internação pediátrica em questão apresenta a particularidade de estar inserida em um hospital escola, recebendo alunos de diversas áreas. Essa situação acarreta no ambiente de trabalho com movimento constante de diferentes profissionais, podendo interferir na comunicação, de modo a gerar a perda de informações fundamentais para a continuidade do cuidado, com repercussões negativas para a segurança do paciente pediátrico. Por isso, torna-se necessária à implementação de barreiras 
para interferir nesses fatores que prejudicam a comunicação eficaz.

Os profissionais da equipe de enfermagem têm o compromisso de garantir a segurança da criança e do adolescente hospitalizado. Nesse sentido, o cuidado e o ambiente estão interligados e devem ser foco de atenção de toda a equipe, uma vez que as delimitações de espaços podem influenciar no processo efetivo da comunicação.

Para a realização da passagem de plantão de forma adequada, o ambiente deve ser tranquilo, espaçoso, ventilado, iluminado, com cadeiras ou bancos para que os profissionais possam realizar essa atividade com o mínimo de interrupções possíveis. ${ }^{(10)}$

As falas dos entrevistados apontam para possíveis causas de perda de informações durante a passagem de plantão entre as equipes de saúde, entendendo-se que essas causas são multifocais, por isso os profissionais devem traçar estratégias que visem minimizar os fatores que interferem negativamente na comunicação durante a passagem de plantão e torná-la mais segura e efetiva.

A comunicação na passagem de plantão sob as condições adversas, como barulhos, imagens e ruídos, pode levar a equipe a cometer erros e estes ocorrem em função das interrupções que afetam a capacidade de concentração do profissional.

Os prejuízos da comunicação durante a passagem de plantão estão diretamente relacionados às barreiras existentes no ambiente, como as interrupções e os ruídos frequentes que inviabilizam a clareza da informação transmitida. Em relação a esse aspecto, um estudo que teve como objetivo examinar o padrão de comunicação entre médicos e enfermeiros verificou que um terço das situações de comunicação era interrompido (uma média de 15 interrupções por hora) e que, na maioria dos casos, os profissionais demoravam a retomar ao assunto interrompido. ${ }^{(11)}$

A falta de orientações sobre as normas e rotinas da unidade pediátrica, assim como a comunicação ineficaz entre profissionais e o acompanhante da criança e adolescente hospitalizada, pode gerar situações de conflito. Os sentimentos de frustração e a ansiedade são minimizados quando o ambiente se torna familiar e os acompanhantes têm as informações sobre o que podem esperar da instituição e sobre o que é esperado deles.
A hospitalização infantil leva os pais a manifestarem sentimentos de medo, ansiedade e frustração. O medo e a ansiedade podem estar relacionados com a gravidade da própria doença e com os tipos de procedimentos envolvidos no tratamento. Já a frustração está frequentemente relacionada com o fato de não se sentirem bem-vindos pela equipe, com o desconhecimento de normas e rotinas da instituição e com a falta de informação acerca de procedimentos e tratamentos. ${ }^{(12)}$

Podemos constatar que existem inúmeros fatores que interferem na comunicação, e frente a isso a passagem de plantão é um recurso estratégico para a organização do cuidado em uma unidade de internação, sendo que as interrupções podem comprometer a qualidade das informações transmitidas e a garantia da continuidade dos cuidados aos pacientes. ${ }^{(10)}$

\section{Barreiras relacionadas à participação dos funcionários na passagem de plantão}

O trabalho dos profissionais de enfermagem em uma unidade hospitalar implica o cumprimento de regras de cunho trabalhista, ético, regimental e legal. Em relação à passagem de plantão, a sua estrutura, muitas vezes, não está descrita de forma objetiva, sistemática e direta em alguns hospitais. ${ }^{(13)}$

A busca incessante pela melhoria de suas condições de vida e subsistência gera inevitavelmente um esgotamento físico, psíquico e intelectual dos profissionais, o que faz com que estes deixem de ter compromisso com o que fazem e com a segurança do paciente pediátrico. $\mathrm{O}$ atraso de membros da equipe, a entrada e saída de funcionários, as saídas apressadas dos profissionais e a impontualidade para o início da atividade são comuns durante a passagem de plantão. ${ }^{(14)}$

A passagem de plantão vem imbuída de uma série de responsabilidades, entre elas a responsabilidade civil, entretanto não se observa delineada em nenhuma lei, sequer no Código de Ética do Profissional da Enfermagem ou na Lei do Exercício Profissional. ${ }^{(13)}$

A aparente ausência de normas que rejam a passagem de plantão indicam que as lacunas que cercam o assunto podem ocasionar danos para o paciente, profissionais e instituição. A normatização da passagem de plantão não é padronizada e nem vem amparada em qualquer lei que se relacione ao exercício profis- 
sional ou ao contrato de trabalho, mas é amplamente cobrada no momento da execução das atividades.

No Código de Ética do Conselho Federal de Enfermagem, no artigo 16, lê-se: "[...] garantir a continuidade da assistência de enfermagem em condições que ofereçam segurança, mesmo em caso de suspensão das atividades profissionais decorrentes a movimentos reivindicatórios da categoria"..(15)

A passagem de plantão torna-se ponto frágil pelo simples fato de não haver padronização ou regramento legal que estabeleça direitos e obrigações perante a possibilidade de falhas e por não estar delimitada nos contratos de trabalho de forma a não violar a capacidade laborativa desses profissionais e assegurar a segurança dos pacientes. ${ }^{(13)}$

O processo da passagem de plantão demanda comprometimento, atenção e envolvimento, pois os profissionais de enfermagem serão os responsáveis para que a comunicação ocorra de forma eficaz. Cabe a estes profissionais mudar suas atitudes e a maneira de perceber esse momento, procurando estabelecer um método interativo, educativo, de comprometimento junto aos demais profissionais, para que haja ampla participação de todos os envolvidos, trazendo como resultados a continuidade e a segurança nos cuidados prestados àqueles que necessitam da qualidade no cuidado prestado, ou seja, a criança, o adolescente e sua família.

Ainda, segundo o parecer técnico do Conselho Regional de Enfermagem de São Paulo, a passagem de plantão constitui uma fase do processo de trabalho da enfermagem; não realizá-la, constitui-se em negligência, tanto para quem finda a atividade de um turno quanto para quem inicia o turno, caracterizando infração ética. ${ }^{(16)}$

\section{Conclusão}

A comunicação eficaz na passagem de plantão requer a superação das barreiras de ordem do ambiente e humana. $\mathrm{O}$ ambiente físico precisa ser adequado, com diminuição de ruídos e de eventuais interferências. Além disso, a passagem de plantão deve ser realizada com seriedade pelos profissionais de enfermagem, no que diz respeito principalmente à sua efetiva participação, transmitindo informações relevantes para a continui- dade do cuidado e a segurança do paciente pediátrico. Na pretensão de minimizar os fatores que interferem na comunicação na passagem de plantão, faz-se necessária a sensibilização dos profissionais para o desenvolvimento de estratégias que amplifiquem os olhares sobre o que interfere na comunicação e principalmente implementar barreiras de segurança, trazendo como resultado uma comunicação eficaz na passagem de plantão, considerando que essa atividade contribui para a continuidade do cuidado. Entende-se que a comunicação eficaz só se concretiza ou acontece quando se transcendem as barreiras que dificultam o desempenho de um processo importante entre as atribuições dos profissionais de enfermagem. Cabe ao enfermeiro gerenciar, destacar e procurar solucionar junto com os demais profissionais de enfermagem formas de minimizar essas barreiras, tornando a comunicação um diferencial para a segurança do paciente pediátrico.

\section{Contribuições}

Silva MF, Anders JC, Rocha PK, Tomazoni A e Scapin SQ declaram que contribuíram com a concepção do estudo, análise e interpretação dos dados, redação do artigo, revisão crítica relevante do conteúdo intelectual e aprovação da versão final a ser publicada.

\section{Referências}

1. Manser T, Foster S. Effective handover communication: an overview of research and improvement efforts. Best Pract Res Clin Anaesthesiol. 2011;25(2):181-91.

2. WegnerW, Pedro EN.A segurança do paciente nas circunstâncias de cuidado: prevenção de eventos adversos na hospitalização infantil. Rev Lat Am Enfermagem. 2012;20(3):427-34.

3. Horwitz LI, Meredith T, Schuur JD, Shah NR, Kulkarni RG, Jenq GY. Dropping the baton: a qualitative analysis of failures during the transition from emergency department to inpatient care. Ann Emerg Med. 2009;53(6):701-10.

4. World Health Organization. World Alliance for Patient Safety. Forward Programme. Geneva: WHO; 2008.

5. Bohmol E. Participação da família na segurança do paciente. In: Harada MJ, organizadores. Gestão em enfermagem: ferramentas para prática segura. São Caetano do Sul: Ed Yendis; 2011. p. 295-304.

6. Minayo MC. 0 desafio do conhecimento: pesquisa qualitativa em saúde. $11^{\mathrm{a}}$ ed. São Paulo: Hucitec; 2008.

7. Santana JC, Sousa MA, Soares HC, Avelino KS. Fatores que influenciam e minimizam os erros na administração de medicamentos pela equipe de enfermagem. Enfermagem Revista. 2012;15(1):122-37.

8. Organização Mundial da Saúde. Ambientes de trabalho saudáveis: um modelo para ação: para empregadores, trabalhadores, formuladores de política e profissionais. Brasília: 0MS; 2010.

9. Pedreira ML. Enfermagem para a segurança do paciente. Acta Paul Enferm. 2009;22(4):56. 
Fragilidades da passagem de plantão em uma unidade pediátrica na perspectiva da equipe de enfermagem

10. Portal KM, MagalhãesAM. Passagem de plantão um recurso estratégico para a continuidade do cuidado em enfermagem. Rev Gaúcha Enferm. 2008;29(2):246-53.

11. Spencer J, Loris N. Three Mile Island and Chernobyl: what went wrong and why today's reactors are safe. WebMemo. 2009;2(6):138-42.

12. Hockenberry M, Wilson DW. Fundamentos de Enfermagem Pediátrica. $9^{\mathrm{a}}$ ed. Rio de Janeiro: Elsevier; 2014.

13. Brasileiro F0, Dias CA. Responsabilidade civil do enfermeiro na passagem de plantão hospitalar. Rev. Eletrônica do Inst. Sergipano Direito Estado. 2012;1(4):98.
14. Silva EE, Campos LF. Passagem de plantão na enfermagem: revisão da literatura. Cogitare Enferm. 2007;12(4):502-7.

15. Conselho Federal de Enfermagem. Resolução COFEN n ${ }^{0}$. 311, de 08 de fevereiro de 2007. Aprova a Reformulação do Código de Ética dos Profissionais de Enfermagem. Diário Oficial da União, Brasília (DF); 2007 Fev 8 [citado 2011 Ago 25]. Disponível em: http://www. cofen.gov.br/resoluo-cofen-3112007_4345.html

16. Conselho Regional de Enfermagem de São Paulo (COREN-SP). Anotações de enfermagem. São Paulo: COREN-SP; 2011. 